\title{
Serum levels of soluble adhesion molecules in newly diagnosed acute myeloid leukemia and in complete remission suggest endothelial cell activation by myeloblasts
}

\author{
Tomas Kupsaa, , Jan Vanekc, Pavel Zak ${ }^{\mathrm{b}, \mathrm{d}}$, Ladislav Jebavy ${ }^{\mathrm{a}, \mathrm{b}}$, Jan M. Horacek ${ }^{\mathrm{a}, \mathrm{b}}$
}

\begin{abstract}
Background and Aims. Despite high-dose multi-agent chemotherapy and allogeneic stem cell transplantation, the relapse rate of acute myeloid leukemia (AML) is high. Further, the disease is highly resistent to drugs. We speculated that deeper understanding of AML-endothelial cell interactions might provide new targets for selective modulation of the AML microenvironment and form the basis for novel treatment approaches. In this study, we evaluated levels of endothelium derived soluble adhesion molecules in active disease and in complete remission (CR) and their relationship with inflammatory cytokines.

Methods. Baseline serum levels of 25 cytokines and 5 soluble adhesion molecules were measured in 84 AML patients using biochip array technology. CR samples were evaluated in 44 patients of this cohort. The control group consisted of 15 healthy blood donors.

Results. All analytes were independent of age or disease origin. Some correlations were restricted to active AML, some were ubiquitous and some were found in remission. In active disease, E-selectin (E-SEL) and VCAM-1 correlated with leukocyte count, E-SEL correlated with P-selectin (P-SEL). Platelet count related to IL-7, EGF and VEGF but not to P-SEL. In CR, P-SEL correlated with platelet count and EGF but not with E-SEL. There was no relationship of P-SEL and E-SEL in the control group.

Conclusions. Leukemic activity is associated with a different pattern of soluble adhesion molecule levels. Both E-SEL and P-SEL may be derived from endothelial cells. Their levels correlated in active disease. E-SEL correlated with leukocyte count. In CR, P-SEL physiologically correlated with platelet count. The correlation with E-SEL was insignificant and absent in the control group. Our data suggest activation of endothelial cells in the presence of myeloblasts.
\end{abstract}

Key words: acute myeloid leukemia, cytokines, adhesion molecules, endothelial cell, complete remission

Received: July 23, 2016; Accepted: October 21, 2016; Available online: November 10, 2016 https://doi.org/10.5507/bp.2016.054

${ }^{a}$ Department of Military Internal Medicine and Military Hygiene, University of Defence, Faculty of Military Health Sciences (FMHS), Hradec Kralove, Czech Republic

${ }^{b} 4^{\text {th }}$ Department of Internal Medicine - Hematology, University Hospital Hradec Kralove, Czech Republic

'Department of Informatics and Quantitative Methods, Faculty of Informatics and Management, University of Hradec Kralove, Hradec Kralove, Czech Republic

${ }^{d} 4^{\text {th }}$ Department of Internal Medicine - Hematology, Faculty of Medicine, Charles University in Prague, Hradec Kralove, Czech Republic Corresponding author: Jan M. Horacek, e-mail:jan.horacek@unob.cz

\section{INTRODUCTION}

The outcome of acute myeloid leukemia (AML) treatment is beleaguered by the high resistance of malignant clones to drugs. Cytokines and adhesion molecules have been studied as markers of immune system activation in many diseases including AML. Soluble (s) forms of adhesion molecules arise from proteolytic cleavage of surface-expressed molecules ${ }^{1}$. In an earlier publication, we described the correlation of inflammatory cytokines such as tumour necrosis factor- $\alpha($ TNF- $\alpha)$ and soluble adhesion molecule levels and our data suggested a critical role of endothelial cell activation on this process. As activated endothelial cells are a source of both platelet selectin (P-SEL) and endothelial selectin (E-SEL) and, platelets are a natural source of P-SEL (ref. ${ }^{2}$ ), we were challenged to include changing parameters of blood count associated with remission into our statistical analyses. Experimentally, leukemic cytokines enhance endothelial cell growth in a co-culture of AML and endothelial cells ${ }^{3}$. AML cells were found to trigger positive feedback loops and activate endothelial cells. Activated endothelial cells express the adhesion molecules involved in leukocyte rolling (P-SEL and E-SEL), leukocyte adhesion (VCAM-1, ICAM-1), chemo attractants MCP-1 and IL-8 (ref., ${ }^{4,5}$ ). Endothelial cell activation by TNF- $\alpha$ was found to be followed by concomitant induction of VCAM-1, ICAM-1 and E-SEL expression and time-dependent increase in the number of myeloblasts attached to the endothelium ${ }^{6}$. These interactions form the molecular basis of life threatening leukostasis and extramedullary spread of AML. Further, adhesion to activated endothelia may dramatically increase the resistance of AML to chemotherapy and negatively influence treatment outcome ${ }^{7}$. In sum, AML cell susceptibility to chemotherapy is modulated by 
dynamic adhesive interactions. To provide more evidence of these interactions, we analysed the serum levels of a broad panel of cytokines, cytokine receptors, matrix metalloproteinase-9 and soluble adhesion molecules. We compared newly diagnosed (active) AML with patients in complete remission (CR). A control group of 15 healthy blood donors was used to confirm the most important findings in CR.

Despite high-dose multi-agent chemotherapy and allogeneic stem cell transplantation, the relapse rate is high and the same is true for the toxicity of commonly used high-dose treatment regimens.

We speculated that deeper understanding of AMLendothelial cell interactions might provide new targets for selective modulation of the AML microenvironment and form the basis for novel treatment approaches.

\section{PATIENTS AND METHODS}

\section{Patients}

A total of 84 newly diagnosed AML patients were studied in the period 2010 - 2015. Further details on the patient cohort are summarized in Table 1. The secondary AML patient group consisted of 32 patients with progression from myelodysplastic syndrome $(n=21)$, myeloproliferative diseases $(n=5)$, chronic myelomonocytic leukemia $(n=3)$ and the blastic phase of chronic myeloid leukemia $(n=3)$. Only patients eligible for induction chemotherapy were included. All were tested for karyotype and molecular genetic aberrations. Twenty-three patients were Famosin-like tyrosin kinase 3 - internal tandem duplication (FLT3-ITD) positive, mutation in NPM-1 occurred in 25 cases. Seven patients had acute promyelocytic leukemia (APL). Risk stratification was carried out according to the European Leukemia Net (ELN) recommendations $^{8}$. Initial hyperleukocytosis (Leucocyte count $\geq 50 \times 10^{9} / \mathrm{L}$ or clinical signs of leukostasis) requiring leukapheresis was present in 23 cases. Patients with APL were treated with the PETHEMA regimen combining Idarubicin and All-trans retinoic acid (ATRA). All other patients were induced with " $3+7$ " induction chemotherapy consisting of Cytarabin $100 \mathrm{mg} / \mathrm{m}^{2}$ per day for 7 consecutive days and Daunorubicin $90 \mathrm{mg} / \mathrm{m}^{2}$ for the first 3 days of therapy in younger patients. Since the beginning of 2015, the induction dose of Daunorubicin used has been $60 \mathrm{mg} / \mathrm{m}^{2}$ even in younger patients, according to recent evidence-based data modifications. In patients aged $\geq 65$ years, Daunorubicin $45-60 \mathrm{mg} / \mathrm{m}^{2}$ was administered, depending on patient WHO performance status. Nine patients had initial infection requiring intravenous antibiotic treatment. The study was approved by the local Ethics Committee and carried out in accordance with the current Helsinki Declaration. All patients gave their written consent to the study.

\section{Complete remission samples}

CR was evaluated according to NCCN criteria. A total of 45 patients were enrolled for evaluation in CR, 30 with primary AML and 15 with secondary AML. To ascertain deeper remission, all samples were obtained after at least 2 cycles of chemotherapy. Originally, all patients were intended for evaluation at CR but in 4 cases we failed to obtain a CR sample. Further shrinkage of the cohort was caused by disease progression or death in aplasia $(n=8)$, voluntary discontinuation of treatment $(n=1)$, intolerance to the treatment with further cytoreductive and palliative approach (low-dose cytarabin or hydroxyurea, $\mathrm{n}=4$ ) and a programme of sequence allogeneic stem cell transplantation ongoing on our department. In 3 cases of chemosensitivity, these patients did not meet the inclusion criteria (CR and after at least 2 cycles of chemotherapy) before final laboratory measurements. The cohort of 45 patients evaluated at CR thus comprised patients who were indicated for allogeneic stem cell transplantation. These underwent induction chemotherapy and at least 1 cycle of either consolidation or salvage therapy. The same was true for those who were not indicated for allogeneic stem cell transplant owing to low risk of AML, higher age or comorbidities.

Twenty-four patients had a molecular genetic marker of minimal residual disease (MRD). Of these, fifteen were MRD-negative at pair serum collection. In 21 cases, the MRD was evaluated using 8 colour flow cytometry. MRD negativity by flow cytometry was attained in $11 / 21$ cases. One patient with unclassified myeloproliferative disorder had a platelet count of $711 \times 10^{9} / \mathrm{L}$ and positive MRD by flow cytometry. The depth of remission (or residual myeloproliferative changes in bone marrow) was not evaluated by trephine biopsy. Due to high risk of bias, this sample was excluded from further analyses. Patients after allogeneic stem cell transplantation were not included in the evaluation in CR.

\section{Serum collection}

Peripheral blood was collected in serum using separating tubes, immediately transported to the laboratory and processed within $2 \mathrm{~h}$ of collection by centrifugation at $1500 \mathrm{rpm}$ x $5 \mathrm{~min}$. All sera were collected before leukapheresis, administration of hydroxyurea or induction therapy. If specimens were not to be analysed immediately, they were stored frozen in small aliquots at below -70 ${ }^{\circ} \mathrm{C}$ as recommended by the Cytokine Array manufacturer. Repeat freeze/thaw cycles were avoided.

\section{Biochip array technology}

All analytes were measured by biochip array technology using chemiluminescent sandwich immunoassays applied to the Evidence Investigator analyzer (Randox Laboratories Ltd., Crumlin, UK). The Evidence Investigator biochip array technology is used to perform simultaneous quantitative detection of multiple analytes from a single patient sample. The core technology is the Randox biochip, a solid-state device containing an array of discrete test regions of immobilised antibodies, specific to different cytokines and growth factors. A sandwich chemiluminescent immunoassay was employed for the cytokine array. Increased levels of cytokine in a specimen will lead to increased binding of antibody labelled with horseradish peroxidase (HRP) and thus an increase 
in the chemiluminescent signal emitted. The light signal generated from each of the test regions on the biochip is detected using digital imaging technology and compared to that from a stored calibration curve. The concentration of analyte present in the sample is calculated from the calibration curve.

We evaluated circulating levels of the following factors: interleukins (IL-1 $\alpha$, IL-1 $\beta$, IL-2, IL-3, IL-4, IL-5, IL-6, IL-7, IL-8, IL-10, IL-12p70, IL-13, IL-15, IL-23), epidermal growth factor (EGF), granulocyte-macrophage colony stimulating factor (GM-CSF), interferon-gamma (IFN- $\gamma$ ), monocyte chemotactic protein-1 (MCP-1), macrophage inflammatory protein- $1 \alpha$ (MIP-1 $\alpha)$, matrix-metalloproteinase-9 (MMP-9), tumour necrosis factor-alpha (TNF- $\alpha$ ), vascular endothelial growth factor (VEGF), soluble IL-2 receptor- $\alpha$ (sIL-2R $\alpha)$ and soluble receptors for IL-6 (sIL-6R) and TNF- $\alpha$ type I and II (TNFR1,2), E-selectin (E-SEL), P-selectin (P-SEL), L-selectin (L-SEL), intercellular adhesion molecule-1 (ICAM-1) and vascular cell adhesion molecule-1 (VCAM-1). The results are expressed in nanograms per litre $(\mathrm{ng} / \mathrm{L})$ for cytokines and micrograms per litre $(\mu \mathrm{g} / \mathrm{L})$ for adhesion molecules, soluble cytokine receptors and MMP-9.

\section{Leukemia-negative controls}

The control group consisted of 15 healthy blood donors (median age 41, range 25-58 years, 11 males). All were free of clinical signs of infection prior to serum collection, which was immediately before peripheral blood sampling.

\section{Statistical analysis}

Statistical analysis was performed in the R statistical programming environment, version 3.1.2 (http://www.Rproject.org). The levels of L-SEL exceeded upper limit of the Array sensitivity in the majority of patients with active AML, but did not exceed this limit in CR samples. The levels of IL-23 were below the threshold limits for the majority of patients and hence L-SEL and IL-23 were not included in further analyses. We calculated the correlations between cytokine levels, soluble adhesion molecules and age, leukocyte count, haemoglobin levels, platelet count, C-reactive protein (CRP) levels, albumin, lactic dehydrogenase (LDH), APL, secondary AML, presence of BCR/ABL, complex karyotype, FLT3-ITD and mutation in NPM-1. Spearman correlations were used pair-wise for continuous variables. To test the association between dichotomous and continuous variables the Mann-Whitney U test was used. Fisher's exact test was used for pairs of categorical variables. The Bonferroni-Holm correction for multiple testing was applied where necessary. In all analyses, $P=0.05$ was considered statistically significant.

\section{RESULTS}

\section{Influence of age, AML origin and genetic aberrations}

The levels of IL-5 were higher in patients younger than 65 years but the difference was not significant after Bonferroni-Holm correction of $P$ value. The patients with secondary AML were older $(P=0.0175)$ and in these patients the FAB M2 subtype of AML was more frequent $(P=0.0327)$. We found no other significant differences in evaluated analytes associated with age.

Normal karyotype had a close relationship with primary AML $(P=0.0063)$. The patients with normal karyotype AML had higher leukocyte count, which was not significant after Bonferroni-Holm correction, corrected $(P=0.2356)$. Secondary AML was significantly associated with complex karyotype $(P=0.0383)$. There was no association of evaluated analytes with primary or secondary origin of the disease, karyotype, FAB subtype or ELN risk stratification. The FLT3-ITD positive cases had lower IL-7 levels (corrected $P=0.0049)$. The FLT3-ITD positive cases had higher E-SEL (nominal $P=0.0225$ ), which was not significant after Bonferroni-Holm correction (corrected $P=0.2677$ ). No other associations of molecular genetic aberration with any evaluated analyte were observed. The FLT3-ITD positive cases were associated with normal karyotype $(P=0.0074)$ and NPM-1 $(P=0.0025)$. The NPM-1 mutation was associated with primary AML $(P=0.0013)$, normal karyotype $(P=0.0001)$ and higher $\mathrm{CR}$ rates after induction therapy $(P=0.0261)$. Baseline hyperleukocytosis was more frequent in NPM-1 mutated cases $(P=0.0083)$.

\section{Factors associated with blood count and adhesion molecule} levels in active AML

The leukocyte count correlated with E-SEL ( $\mathrm{r}=$ $0.7572, P=0.0001)$, VCAM-1 $(\mathrm{r}=0.5495, P=0.0006)$, TNFR-1 ( $\mathrm{r}=0.6143, P=0.0001)$, TNFR-2 $(\mathrm{r}=0.5919, P=$ $0.0001), \mathrm{LDH}(\mathrm{r}=0.6773, P=0.0001)$ and negatively with IL-7 ( $\mathrm{r}=-0.4705, P=0.0271)$. The patients with initial hyperleukocytosis had much higher levels of E-SEL than other patients $(P=0.0002)$. The haemoglobin levels were generally independent of other variables. Six variables had at least weak correlation with haemoglobin levels with absolute values of " $\mathrm{r}$ " ranging from 0.2012 to 0.3663 , but none of them was statistically significant. The platelet count correlated with IL-7 $(\mathrm{r}=0.6845, P=0.0001)$, EGF (r $=0.7060, P=0.0001)$ and VEGF ( $\mathrm{r}=0.5211$, $P=0.0025)$. The EGF levels correlated with VEGF $(\mathrm{r}=$ $0.4615, P=0.0396$ ). The levels of soluble adhesion molecules correlated. Their correlations with other factors are summarized in Table 2.

\section{Inflammatory markers and other correlations in active AML}

The CRP levels correlated strongly with IL-6 ( $\mathrm{r}=$ $0.7095, P \quad 0.0001)$, ICAM-1 $(P=0.0122)$ and negatively with albumin levels $(\mathrm{r}=-0.4806, P=0.0175)$. Correlations of IL-6 with ICAM-1 and TNFR-1were close to statistical significance $(\mathrm{r}=0.4496, P=0.0646)$ and $(\mathrm{r}=0.4438, P=$ $0.0815)$ respectively. The IL-1 $\alpha$ correlated with IL-2 $(\mathrm{r}=$ $0.4556, P=0.0506)$ and IL-4 $(\mathrm{r}=0.5039, P=0.0059)$. The levels of TNF- $\alpha$ correlated positively with TNFR-2 ( $\mathrm{r}=0.5377, P=0.0011)$, but not with TNFR-1 ( $\mathrm{r}=0.3004$, corrected $P=1)$. TNFR-2 correlated with TNFR-1 $(\mathrm{r}=$ $0.6442, P=0.0001)$ and sIL-6R $(\mathrm{r}=0.5721, P=0.0001)$. IL-3 correlated with IL-12 $(\mathrm{r}=0.4624, P=0.0383)$, IL- 
Table 1. Baseline characteristics of studied group.

\begin{tabular}{|c|c|c|c|}
\hline & All patients & Primary AML & Secondary AML \\
\hline Patients & 84 & $52(62 \%)$ & $32(38 \%)$ \\
\hline Age (years) & $53.3 \pm 12.2$ & $49.3 \pm 12.3$ & $59.8 \pm 9.2$ \\
\hline \multicolumn{4}{|l|}{ Sex } \\
\hline Male & $34(40 \%)$ & $22(42 \%)$ & $12(37 \%)$ \\
\hline Female & $50(60 \%)$ & $30(58 \%)$ & $20(63 \%)$ \\
\hline \multicolumn{4}{|l|}{ Cytogenetics (risk) } \\
\hline Low & 18 & 17 & 1 \\
\hline Intermediate & 29 & 18 & 11 \\
\hline High & 37 & 17 & 20 \\
\hline $\begin{array}{l}\text { Leucocyte count }\left(\times 10^{9} / \mathrm{L}\right) \text {, } \\
\text { median }\end{array}$ & $\begin{array}{c}50.7 \pm 57.3 \\
14.9\end{array}$ & $\begin{array}{l}48.1 \pm 50.1 \\
16.4\end{array}$ & $\begin{array}{c}54.8 \pm 69.4 \\
7.8\end{array}$ \\
\hline \multicolumn{4}{|l|}{ FAB type } \\
\hline M0 & 8 & 3 & 5 \\
\hline M1 & 17 & 11 & 6 \\
\hline M2 & 32 & 16 & 16 \\
\hline M3 & 7 & 7 & 0 \\
\hline M4 & 12 & 8 & 4 \\
\hline M5 & 7 & 7 & 0 \\
\hline M7 & 1 & 0 & 1 \\
\hline Active infection & $9(11 \%)$ & $4(8 \%)$ & $5(16 \%)$ \\
\hline $\mathrm{CRP}(\mathrm{mg} / \mathrm{L})$ & $47.1 \pm 48.4$ & $39.3 \pm 37.1$ & $59.0 \pm 68.2$ \\
\hline FLT3-ITD & $23(21 \%)$ & $18(35 \%)$ & $5(16 \%)$ \\
\hline NPM-1 mutated & $25(30 \%)$ & $22(42 \%)$ & $3(9 \%)$ \\
\hline BCR-ABL & $3(4 \%)$ & 0 & $3(9 \%)$ \\
\hline Complex karyotype & $10(12 \%)$ & $3(6 \%)$ & $7(22 \%)$ \\
\hline
\end{tabular}

Table 2. Correlations of soluble adhesion molecule levels with other factors in active AML.

\begin{tabular}{|c|c|c|c|c|c|c|c|c|}
\hline \multirow[t]{2}{*}{ Analyte } & \multicolumn{2}{|c|}{ VCAM-1 } & \multicolumn{2}{|c|}{ ICAM-1 } & \multicolumn{2}{|c|}{ E-SEL } & \multicolumn{2}{|c|}{ P-SEL } \\
\hline & $\mathrm{r}$ & $P$ & $\mathrm{r}$ & $P$ & $\mathrm{r}$ & $P$ & $\mathrm{r}$ & $P$ \\
\hline VCAM-1 & & & 0.5993 & $<0.0001$ & 0.5363 & 0.0011 & 0.4406 & 0.0922 \\
\hline ICAM-1 & 0.5993 & $<0.0001$ & - & & 0.4693 & 0.0285 & 0.2058 & 1 \\
\hline E-SEL & 0.5363 & 0.0011 & 0.4693 & 0.0285 & - & & 0.6087 & $<0.0001$ \\
\hline P-SEL & 0.4406 & 0.0922 & 0.2058 & 1 & 0.6087 & $<0.0001$ & - & \\
\hline Leucocyte count & 0.5495 & 0.0006 & 0.4171 & 0.2251 & 0.7572 & $<0.0001$ & 0.4407 & 0.0918 \\
\hline LDH & 0.6005 & $<0.0001$ & 0.4649 & 0.0344 & 0.7545 & $<0.0001$ & 0.4499 & 0.0640 \\
\hline CRP & 0.0249 & 1 & 0.4885 & 0.0122 & 0.0980 & 1 & -0.2883 & 1 \\
\hline IL-6 & 0.1856 & 1 & 0.4496 & 0.0646 & 0.1036 & 1 & -0.1999 & 1 \\
\hline $\mathrm{TNF} \alpha$ & 0.6474 & $<0.0001$ & 0.4288 & 0.1453 & 0.3468 & 1 & 0.3445 & 1 \\
\hline TNFR-1 & 0.5086 & 0.0047 & 0.5466 & 0.0007 & 0.4837 & 0.0152 & 0.2455 & 1 \\
\hline TNFR-2 & 0.6076 & $<0.0001$ & 0.4431 & 0.0835 & 0.4773 & 0.0202 & 0.2835 & 1 \\
\hline sIL-2R $\alpha$ & 0.4749 & 0.0224 & 0.2189 & 1 & 0.1835 & 1 & 0.1821 & 1 \\
\hline sIL-6R & 0.4733 & 0.0240 & 0.2507 & 1 & 0.3612 & 1 & 0.2539 & 1 \\
\hline MMP-9 & 0.3545 & 1 & 0.0545 & 1 & 0.4114 & 0.2769 & 0.4842 & 0.0149 \\
\hline
\end{tabular}

r - Spearman correlation coefficient

$P$ - Bonferroni-Holm corrected values for multiple testing

$13(\mathrm{r}=0.5396, P=0.0010)$ and GM-CSF $(\mathrm{r}=0.4527$, $P=0.0570)$. The levels of IL-12 and IL-13 correlated $(\mathrm{r}=0.5038, P=0.0060)$. GM-CSF correlated with IL-5 $(\mathrm{r}=0.6419, P=0.0001)$. No other correlations were statistically significant.

\section{Changes of evaluated analytes in complete remission}

Achieving CR was associated with normalisation of leukocyte count and platelet levels, improved haemoglo- bin levels, normalisation of LDH, CRP and better albumin levels. In CR, the levels of EGF, VEGF and IL-7 were higher than in active AML. In contrast, the levels of IL-6, IL-8, sIL-6R and TNFR-1 were lower in CR. All these changes are summarised in Table 3 . The increase in CR levels of P-SEL and MMP-9, same as the decrease of E-SEL, IL-5, IL-10, IL-15, IFN- $\gamma$, GM-CSF, and sIL-2R $\alpha$ was not significant after $P$ value correction for multiple testing. 
Table 3. Changes associated with CR achievement.

\begin{tabular}{|c|c|c|c|}
\hline Variable & Active AML & Complete remission & $P$ (Bonferroni) \\
\hline Leukocyte count $\left(\times 10^{9} / \mathrm{L}\right)$ & $30.56 \pm 64.66$ & $5.75 \pm 1.84$ & 0.1685 \\
\hline Platelet count $\left(\times 10^{9} / \mathrm{L}\right)$ & $68.67 \pm 33.75$ & $219.82 \pm 89.83$ & $<0.0001$ \\
\hline Haemoglobin $(\mathrm{g} / \mathrm{L})$ & $90.36 \pm 17.80$ & $119.43 \pm 15.59$ & $<0.0001$ \\
\hline Albumin $(\mathrm{g} / \mathrm{L})$ & $40.00 \pm 3.56$ & $43.04 \pm 3.20$ & 0.0032 \\
\hline CRP (mg/L) & $48.24 \pm 49.50$ & $5.01 \pm 3.06$ & 0.0132 \\
\hline LDH ( $\mu$ kat/L) & $8.11 \pm 4.70$ & $3.69 \pm 0.78$ & 0.0002 \\
\hline $\mathrm{EGF}(\mathrm{ng} / \mathrm{L})$ & $11.61 \pm 11.08$ & $49.44 \pm 30.78$ & $<0.0001$ \\
\hline VEGF (ng/L) & $54.50 \pm 30.95$ & $175.16 \pm 85.76$ & $<0.0001$ \\
\hline IL-7 (ng/L) & $5.64 \pm 3.87$ & $26.97 \pm 13.05$ & $<0.0001$ \\
\hline IL-6 (ng/L) & $12.27 \pm 10.94$ & $2.73 \pm 2.18$ & 0.0134 \\
\hline IL-8 (ng/L) & $61.08 \pm 53.17$ & $7.87 \pm 3.39$ & 0.0041 \\
\hline L-SEL $(\mu \mathrm{g} / \mathrm{L})$ & $2494.12 \pm 1015.41$ & $1477.79 \pm 431.65$ & $<0.0001$ \\
\hline sIL-6R $(\mu \mathrm{g} / \mathrm{L})$ & $1.53 \pm 0.77$ & $0.95 \pm 0.34$ & 0.0005 \\
\hline TNFR-1 $(\mu \mathrm{g} / \mathrm{L})$ & $0.60 \pm 0.23$ & $0.40 \pm 0.15$ & 0.0366 \\
\hline
\end{tabular}

Table 4. Summary of correlations of evaluated factors.

\begin{tabular}{lll}
\hline Correlations present at all times & Correlations restricted to active AML & $\begin{array}{l}\text { Correlations } \\
\text { restricted to CR/controls }\end{array}$ \\
\hline VCAM-1, ICAM-1 & E-SEL, P-SEL & Platelet count, P-SEL \\
Platelet count, IL-7, EGF, VEGF & Leukocyte count, E-SEL, VCAM-1, TNFR-1, TNFR-2 & P-SEL, IL-7, (IL-8, EGF, VEGF) \\
IL-1 $\alpha$, (IL-1 $\beta$ ), IL-2, IL-4 & IL-3, IL-12, IL-13 & VEGF, TNF $\alpha$ \\
GM-CSF, IL-5 & & \\
TNFR-2, TNFR-1, sIL-6R & & \\
\hline
\end{tabular}

Cytokines mentioned in brackets correlate with some, but not with all other factors of the group.

\section{Correlation analysis in complete remission}

Leukocyte count correlated negatively with IL-3 ( $\mathrm{r}=$ - $0.6772, P=0.0179)$. There was no correlation of leukocyte count with VCAM-1 $(\mathrm{r}=0.0097)$ and correlation with E-SEL was weak $(r=0.2686)$ and insignificant. Haemoglobin levels correlated with L-SEL ( $r=0.6662$, $P=0.0268)$ and MMP-9 $(\mathrm{r}=0.5185)$. Platelet count correlated with P-SEL $(\mathrm{r}=0.6161)$ and IL-7 $(\mathrm{r}=0.6215)$. VCAM-1 correlated with ICAM-1 $(\mathrm{r}=0.5379)$, TNFR-1 (r $=0.5768, P=0.4218)$ and TNFR-2 $(\mathrm{r}=0.5425, P=$ $0.9925)$. ICAM-1 correlated with TNFR-1 ( $r=0.4837)$. There was no relationship between immunoglobulin and selectin adhesion molecules. The correlation of E-SEL with P-SEL, like the correlations of these adhesion molecules with L-SEL, were weak to moderate but not statistically significant.

\section{Other correlations}

TNFR-2 correlated with TNFR-1 ( $\mathrm{r}=0.7413, P=$ $0.0012)$ and sIL-6R $(r=0.5533)$. P-SEL levels correlated with EGF $(\mathrm{r}=0.5677, P=0.5343)$. VEGF moderately correlated with TNF- $\alpha(r=0.4922)$ and IL-7 $(r=0.4125)$, which were not statistically significant. IL-1 $\alpha$ correlated strongly with IL-1 $\beta(\mathrm{r}=0.6274, P=0.0987)$. Both correlated with IL-2. For IL-1 $\alpha$ the correlation was moderate with $\mathrm{r}=0.5237$, IL-1 $\beta$ correlated strongly with $\mathrm{r}=0.7160$ $(P=0.0037)$. All three cytokines correlated with IL-4 with $\mathrm{r}=0.4819$ for IL- $1 \alpha, \mathrm{r}=0.5938$ for IL- $1 \beta$ and $\mathrm{r}=$ $0.6956(P=0.0088)$ for IL-2. CRP correlated with IL-6 (r $=0.6000, P=0.2235)$ and TNF $\alpha(\mathrm{r}=0.5247)$. Albumin correlated negatively with IL-6 $(r=-0.4118)$ and TNF- $\alpha$ $(\mathrm{r}=-0.5633)$. Leaving an outlier sample with elevated CRP $(45 \mathrm{mg} / \mathrm{L})$ out of the analysis affected the correlation of CRP and TNF- $\alpha\left(r^{\prime}=0.3188\right)$ and correlations with albumin levels. Correlations of other factors were not affected by excluding this sample from of the analysis. GMCSF strongly correlated with IL-5 $(\mathrm{r}=0.7319, P=0.0018)$ and further with IL-1 $\alpha(\mathrm{r}=0.5129)$, but not with IL-3. Correlations of IL-3 with IL-12 and IL-13, like the correlation of IL-12 and IL-13 were weak and not significant.

Cytokines and adhesion molecules in control group samples Compared to CR measures, the levels of IL-4 ( $P=$ $0.0018)$ and GM-CSF $(P=0.0462)$ were higher in the control group. The levels of VCAM-1 $(P=0.0014)$, ICAM-1 ( $P$ $=0.0162)$ and IL-7 $(P=0.0444)$ were lower in the control group than in remission samples. The levels of VCAM1 correlated with ICAM-1 $(r=0.5607)$ and E-SEL $(r=$ 0.5344). E-SEL levels had no relationship with P-SEL ( $\mathrm{r}=$ 0.0626). P-SEL correlated with IL-7 ( $\mathrm{r}=0.6127)$, IL-8 $(\mathrm{r}$ $=0.6425), \operatorname{EGF}(r=0.7614)$ and $\operatorname{VEGF}(r=0.5572)$. The correlations of EGF with IL-7 $(\mathrm{r}=0.8758, P=0.0087)$ and IL- $8(\mathrm{r}=0.8559, P=0.0218)$ were very strong, like the correlation of VEGF and TNF $\alpha(\mathrm{r}=0.8093, P=0.1192)$. IL-7 correlated with IL-8 $(\mathrm{r}=0.6864)$. IL-1 $\alpha$ correlated with IL-4 $(r=0.7739)$ and MCP-1 $(r=0.7224)$. MCP-1 correlated with IFN- $\gamma(\mathrm{r}=0.7765, P=0.3064)$. IL-5 correlated with GM-CSF $(r=0.6766)$ and MIP-1 $\alpha(r=0.7013)$. TNFR-1 correlated with TNFR-2 $(r=0.6115)$ and sIL-6R $(r=0.6351)$. Based on these results, we identified correla- 
tions that are ubiquitous (with slight modifications) and correlations restricted to active disease or on the other hand to CR/healthy controls, see Table 4.

\section{DISCUSSION}

Better understanding of the cancer microenvironment is a sine qua non for development of new treatment approaches. In vitro experiments published so far, like our previous results, demonstrate that cytokines and adhesion molecules form a unique interacting functional network ${ }^{9,10}$. Overexpression of cytokines in acute myeloid leukemia patients declines after chemotherapy or remission. Here we report that simple comparison of cytokine and adhesion molecule levels in active acute leukemia and complete remission may cause misleading conclusions. Correlation analysis provides a plethora of information. Adding blood count and basic inflammatory markers to the analyses was very helpful in revealing the complexity of the system in active AML which is regulated by different principles than in CR.

In newly diagnosed AML, the levels of IL-7, EGF and VEGF correlated with platelet count and E-SEL correlated with P-SEL. The levels of P-SEL increased in CR (which was not significant after $P$ correction for multiple testing) and compared to active AML P-SEL no longer correlated with E-SEL levels, which were decreased. To explain these findings, we formed a hypothesis of two independent simultaneous processes.

First, achievement of CR leads to improvement in baseline thrombocytopenia, which causes significant increase in platelet related cytokines (IL-7, EGF, VEGF). The literature suggests these cytokines are stored in platelet granules ${ }^{11-13}$, which explains their correlation with platelet count. In CR, P-SEL correlates with platelet count, which is probably due to physiological cleavage of surface P-SEL from platelets, and further with IL-8. IL-8 (CXCL8) was independent of other evaluated factors in active disease and rapidly decreased in CR, see Table 3. These findings confirm the viability of using IL- 8 as a marker of leukemic cell burden, reported previously ${ }^{14}$. CR levels of IL-8 were associated with platelet count. Based on the published data, this association may originate from either direct storage of IL-8 in platelets, or indirectly by secretion of IL- 8 by endothelial cells induced by activated platelets $^{15,16}$.

Second, in active AML, the levels of adhesion molecules correlated with leukocyte count, levels of TNF- $\alpha$, TNFR-1 and TNFR-2. ICAM-1 had a relationship with CRP levels, which were elevated in newly diagnosed patients. Myeloblasts are reported to stimulate endothelial cells in vitro ${ }^{6}$. Our data harvested from in vivo analyses are in agreement with in vitro findings. Further, our analysis included platelet count and P-SEL levels. Both E-SEL and P-SEL can be produced by endothelial cells. Here we report that significant correlations of E-SEL and VCAM-1 with leukocyte count and correlation of E-SEL and P-SEL, are the most important findings for confirming activation of endothelial cells in the presence of myeloblasts.
Adhesion receptors and TNF- $\alpha$ secreted by blast cells play central roles in these reactions. The activation of endothelial cell declines in CR. The correlation of P-SEL and E-SEL, present in active AML, was weak and statistically insignificant. In the control group, this correlation was absent, showing possible artifacts in the background of hematopoiesis reconstitution in CR samples after chemotherapy. Further, we found no relation with leukemia origin or subtype. We consider the endothelial cell activation by myeloblasts is a uniform reaction of endothelial cells to inflammatory stimuli originating from AML activity, irrespective of age or AML origin. In this dataset, only FLT3-ITD positive leukemias had lower IL-7 levels than other cases, which were probably not linked to endothelial cell activation. There is no clear association of lower IL-7 levels and the adverse biology of these leukemias and this requires further investigation. We did not include correlations of soluble adhesion molecule levels and absolute blast count, which might be more exact but cannot be applied to CR samples. Adhesion of myeloblasts to marrow stromal cells or activated endothelial cells is the background of resistance to chemotherapy ${ }^{7,17}$. Mechanical changes of activated endothelial cells induced by myeloblasts of leukemic cell lines HL-60 and KG-1 have been reported recently ${ }^{18}$. Thus, blocking AML cell interactions with endothelium or marrow stromal elements may have ameliorating effects for the majority of AML cases.

The origin of elevated CRP levels in this dataset is unclear in the majority of cases. Nine out of 84 patients had clinical signs of infection at AML diagnosis. Infectious complications are frequent in these patients. The baseline laboratory investigations were carried out according to the statutary recommendations of our department. Procalcitonin levels are not routinely measured and leukemic production of IL-6 is frequent in AML (ref. ${ }^{19}$ ). Thus, it was not possible to distinguish elevation in CRP due to occult infection from that caused by myeloblast activity. On the other hand, only IL-6 and ICAM-1 levels were significantly associated with CRP levels in newly diagnosed AML. The decrease in albumin levels in inflammation and its negative correlation with CRP levels is a common pathophysiological event. In our study, higher CRP was commonly found in newly diagnosed cases, and had no significant relationship with the vast majority of evaluated analytes.

Our data show correlations (with slight modifications) that are ubiquitous and correlations restricted to active disease or on the other hand to $\mathrm{CR} /$ healthy controls, see Table 4. Correlations largely influenced by platelet count were in levels of IL-7, EGF, VEGF and P-SEL in remission. We were unable to confirm this in the controls as blood count parameters were not collected at the time of sampling. Still, the strong correlations of P-SEL with IL-7, IL-8 and EGF and very strong correlation of EGF with IL-7 and IL-8 suggest association with platelet count. For this reason, we consider adding of control group into analyses worth even without exact blood count analysis in these subjects.

In $\mathrm{CR}$, the leukocyte count strongly negatively correlated with IL-3 levels. We consider the above correlation 
quite surprising. IL-3 stimulates the differentiation of multipotent hematopoietic stem cells into myeloid progenitor cells or, with the addition of IL-7, into lymphoid progenitor cells. For this reason we would expect positive correlation if any. To verify this finding, analysis in durable complete remission (at circa 6 months after last chemotherapy in patients not treated with allogeneic stem cell transplantation) or analysis of a sufficient control group including blood count parameters should be performed.

The L-SEL, despite exceeding the upper limit of array sensitivity in the majority of AML samples, significantly decreased in CR. In CR, it strongly correlated with haemoglobin levels. L-SEL is the smallest of selectin adhesion molecules and acts as a "homing receptor" for lymphocytes to enter secondary lymphoid tissues via high endothelial venules. Despite meticulous effort, we found no evidence of a relationship between soluble L-SEL and haemoglobin levels in healthy individuals. The literature describes elevated L-SEL levels following exertion in sickle cell trait carriers ${ }^{20}$, but this article was not aimed at correlation of haemoglobin levels with L-SEL or L-SEL origin. To explain this finding we suggest that L-SEL may non-covalently adhere to erythrocyte surface.

This study was an investigation of the interplay between AML activity and levels of endothelium derived soluble adhesion molecules, which enabled us to analyse their interaction indirectly in vivo. Our data show that endothelial cells are activated in the presence of myeloblasts and this is independent of disease origin or age. The activation declined rapidly in $\mathrm{CR}$ with restoration of normal hematopoiesis. The results and sufficient published data are convincing evidence that targeting these interactions is feasible in the majority of patients and increase AML sensitivity to treatment. The goal of this study was not the prognostic significance of the factors evaluated but such assessment is underway and will be completed in the near future. It was beyond the scope of this study to evaluate the differences in analytes between CR samples and the control group. The latter would provide more evidence for the long-term effects of chemotherapy on human hematopoiesis. Such analyses would require a larger control group of MRD negative patients, preferably in durable $\mathrm{CR}$. We intend to carry out this analysis with a larger cohort of patients and healthy controls.

Acknowledgement: The work was supported by a specific research project "Analysis of defined prognostic factors in acute myeloid leukemia" (FMHS Hradec Kralove), by a long-term organisation development plan 1011 (FMHS Hradec Kralove) and by MH CZ - DRO (UHHK, 00179906).

Author contributions: TK: literature search, research design, statistical analysis, manuscript writing; JV: statistical analysis; PZ, LJ: critical revision; JMH: research design, manuscript writing, critical revision.

Conflict of interest statement: The authors state that there are no conflicts of interest regarding the publication of this article.

\section{REFERENCES}

1. Morandini R, Ghanem G, Portier-Lemarié A, Robaye B, Renaud A, Boeynaems JM. Action of CAMP on expression and release of adhesion molecules in human endothelial cells. Am J Physiol 1996;(7)270:807-16.

2. McEver RP, Beckstead JH, Moore KL, Marshall-Carlson L, Bainton DF. GMP-140, a platelet alpha-granule membrane protein, is also synthesized by vascular endothelial cells and is localized in WeibelPalade bodies. J Clin Invest 1989;84(1):92-9.

3. Hatfield K, Øyan AM, Ersvaer E, Kalland KH, Lassalle P, Gjertsen BT, Bruserud $\varnothing$. Primary human acute myeloid leukaemia cells increase the proliferation of microvascular endothelial cells through the release of soluble mediators. Br J Hematol 2009;144(1):53-68.

4. Scaldaferri F, Sans M, Vetrano S, Correale C, Arena V, Pagano N, Rando G, Romeo F, Potenza AE, Repici A, Malesci A, Danese S. The role of MAPK in governing lymphocyte adhesion to and migration across the microvasculature in inflammatory bowel disease. Eur J Immunol 2009;39(1): 290-300.

5. Zarbock A, Ley K. Neutrophil adhesion and activation under flow. Microcirculation 2009;16(1): 31-42.

6. Stucki A, Rivier AS, Gikic M, Monai N, Schapira M, Spertini O. Endothelial cell activation by myeloblasts: molecular mechanisms of leukostasis and leukemic cell dissemination. Blood 2001;97(7):21219 .

7. Pezeshkian B, Donnelly C, Tamburo K, Geddes T, Madlambayan GJ. Leukemia Mediated Endothelial Cell Activation Modulates Leukemia Cell Susceptibility to Chemotherapy through a positive Feedback Loop Mechanism. Plos One 2013; 8(4):e60823. doi:10.1371/journal. pone.0060823.

8. Döhner H, Estey EH, Amadori S, Appelbaum FR, Büchner T, Burnett AK, Dombret H, Fenaux P, Grimwade D, Larson RA, Lo-Coco F, Naoe T, Niederwieser D, Ossenkoppele GJ, Sanz MA, Sierra J, Tallman MS, Löwenberg B, Bloomfield CD. Diagnosis and management of acute myeloid leukemia in adults: recommendations from an international expert panel, on behalf of the European Leukemia Net. Blood 2010;115(3):453-74.

9. Hsu H, Lee Y, Tsai W, Jiang ML, Ho CH, Ho CK, Wang SY. Circulating levels of thrombopoietic and inflammatory cytokines in patients with acute myeloblastic leukemia and myelodysplastic syndrome. Oncology 2002;63(1):64-9.

10. Kupsa T, Vasatova M, Karesova I, Zak P, Horacek JM. Baseline serum levels of multiple cytokines and adhesion molecules in patients with acute myeloid leukemia: results of a pivotal trial. Exp Oncol. 2014 Dec;36(4):252-7.

11. Shanwell A, Falker C, Gullikson H. Storage of platelets in additive slolutions: the effects of magnesium and potassium on the release of RANTES, beta-thromboglobulin, platelet factor 4 and interleukin-7, during storage. Vox sang 2003;85(3):206-12.

12. Brouwers J, Noviyanti R, Fijnheer R, de Groot PG, Trianty L, Mudaliana $S$, Roest M, Syafruddin D, van der Ven A, de Mast Q. Platelet activation determines angiopoietin-1 and VEGF levels in malaria: Implications for their use as biomarkers. PLoS ONE 2013;8(6):e64850. doi:10.1371/ journal.pone.0064850

13. Burnouf PA, Juan PK, Su CY, Kuo YP, Chou ML, Su CH, Tseng YH, Lin CT, BurnoufT. A novel virally inactivated human platelet lysate preparation rich in TGF-beta, EGF and IGF, and depleted of PDGF and VEGF. Biotechnol Appl Biochem 2010;56(4):151-60.

14. Kittang AO, Hatfield K, Sand K, Reikvam H, Bruserud Ø. The chemokine network in acute myelogenous leukemia: molecular mechanisms involved in leukemogenesis and therapeutic implications. Curr Top Microbiol Immunol 2010;341:149-72.

15. Su SB, Mukaida N, Matsushima K. Rapid secretion of intracellularly pre-stored IL-8 from rabbit platelets upon activation. J Leukoc Biol 1996;59:420-26.

16. Kaplanski G, Porat R, Aiura K, Erban JK, Gelfand JA, Dinarello CA. Activated Platelets Induce Endothelial Secretion of Interleukin-8 In Vitro Via an Interleukin-1-Mediated Event. Blood 1993;81(10): 24925.

17. Matsunaga T, Takemoto N, Sato T, Takimoto R, Tanaka I, Fujimi A, Akiyama T, Kuroda H, Kawano Y, Kobune M, Kato J, Hirayama Y, Sakamaki S, Kohda K, Miyake K, Niitsu Y. Interaction between leukemic-cell VLA-4 and stromal fibronectin is a decisive factor for 
minimal residual disease of acute myelogenous leukemia. Nat Med 2003;9(9):1158-65

18. Tan L, Lin P, Pezeshkian B, Rehman A, Madlambayan G, Zeng X. RealTime Monitoring of Cell Mechanical Changes Induced by Endothelial Cell Activation and their Subsequent Binding with Leukemic Cell Lines. Biosens Bioelectron 2014;56:151-8.

19. Dokter WH, Tuyt L, Sierdsema SJ, Esselink MT, Vellenga E. The spontaneous expression of interleukin-1 beta and interleukin- 6 is as- sociated with spontaneous expression of AP-1 and NF-kappa B transcription factor in acute myeloblastic leukemia cells. Leukemia 1995;9(3):425-32.

20. Tripette J, Connes P, Beltan E, Chalabi T, Marlin L, Chout R, Baskurt OK, Hue O, Hardy-Dessources MD. Red blood cell deformability and aggregation, cell adhesion molecules, oxidative stress and nitric oxide markers after a short term, submaximal, exercise in sickle cell trait carriers. Clin Hemorheol Microcirc 2010;45(1):39-52. 